\title{
Aspergillus fumigatus-specific immunoglobulin levels in BALF of CF patients
}

\author{
To the Editor:
}

A lack of correlation between systemic and local IgE production at mucosal sites has been reported for allergic asthma [1-5], allergic rhinitis [6,7] and chronic rhinosinusitis with nasal polyps [8-10]. In allergic asthmatics, local IgE production is higher than in nonallergic asthmatics [3] and high local IgE levels have been linked to the clinical phenotype. Interestingly, in cases of nasal polyps, local IgE targets mainly superantigens [8].

We recently described elevated levels of the epithelial type 2 cytokine interleukin-33 in the bronchoalveolar lavage fluid (BALF) of patients suffering from cystic fibrosis (CF), which represents a strong upstream signal directly affecting resident innate lymphoid cells, tissue resident eosinophils, mast cells and basophils, as well as Th2 cells [11]. In the lungs of patients with cystic fibrosis, a local Th2- and Th17-biased inflammatory immune milieu with considerable numbers of eosinophils and a combined restrictive and obstructive lung function pattern is observed [12]. Aspergillus fumigatus- and Pseudomonas aeruginosa-specific T-cell lines from CF patients displayed a Th2/Th17 polarisation pattern. Moreover, the rate of allergic sensitisation to A. fumigatus is frequent in CF patients [12]. The relevance of this sensitisation is often unclear and in most of the cases, not linked to the picture of allergic bronchopulmonary aspergillosis (ABPA) [13].

Limited data are available regarding to local immunoglobulin production to A. fumigatus, and its correlation with CF disease progression or lung function. The current study aimed to investigate whether evidence for local immunoglobulin production to A. fumigatus in the lung of CF patients can be found and to what extent this BALF-derived IgE correlates with serum IgE. Moreover, we were interested in the link between A. fumigatus in the lung and local immunoglobulin response. The study was approved by the ethics committee of the Medical University of Vienna, Vienna, Austria (\#081/2007, 355/2009). BALF was obtained from 49 children and adolescents with CF as described recently (median age 6.7 years) [12]. A. fumigatus-specific antibodies (AF-IgE, - IgA and - IgG) were measured in BALF via an "in house" ELISA (Swiss Institute of Allergy and Asthma Research, Davos, Switzerland) [14, 15]. Sensitisation was defined by serum IgE levels against A. fumigatus (ImmunoCAP m3; ThermoFisher, Uppsala, Sweden).

AF-IgE in serum $\left(>0.35 \mathrm{kU} \cdot \mathrm{L}^{-1}\right)$ was observed in $30.6 \%$ of the investigated CF patients. In BALF, $47 \%$ of the patients had AF-IgG, 81.6\% AF-IgA and 32.7\% AF-IgE. Local, lung-derived, A. fumigatus-specific immunoglobulin levels correlated highly with each other (IgA versus IgG: $\mathrm{r}=0.68, \mathrm{p}<0.0001$; IgA versus IgE: $\mathrm{r}=0.66, \mathrm{p}<0.0001$; IgG versus IgE: $\mathrm{r}=0.81, \mathrm{p}<0.0001)$. This suggests that local factors may contribute to immunoglobulin levels in the lung. In contrast, serum AF-IgE and BALF AF-IgE lacked a relevant correlation ( $r=0.24$, not significant). This is in line with studies reporting that allergen stimulation directs IgE production at mucosal sites as the lung or nasal mucosa [2, 4, 8]. Durham et al. [1] were able to show that class switch recombination and somatic hypermutation to IgE occurs locally and independently from serum IgE levels.

To assess the relevance of $A$. fumigatus-specific immunoglobulin levels in BALF and in the lung, subgrouping according to the presence of A. fumigatus in BALF was performed. Local A. fumigatus

$@$ ERSpublications

IgE responses to Aspergillus fumigatus in cystic fibrosis lungs http://ow.ly/XXwv30furqs

Cite this article as: Goña-Höpler M, Pfaller B, Argeny J, et al. Aspergillus fumigatus-specific immunoglobulin levels in BALF of CF patients. ERJ Open Res 2017; 3: 00067-2017 [https://doi.org/ 10.1183/23120541.00067-2017]. 
immunoglobulins have been detected in the BALF of 10 out of 49 CF patients. Specific IgG and IgA levels were significantly elevated in CF patients positive for A. fumigatus compared to CF patients with no evidence for fungal infection $(\mathrm{p}<0.05)$ (figure 1a). AF-IgE levels were higher in CF patients with a positive A. fumigatus culture $(\mathrm{p}=0.09)$. IgA is known to play a critical role in immunity of mucosal sites. It is the most abundant immunoglobulin subtype in secretions as well as in the bronchial mucosa, and functions by binding antigen in order to avoid adherence to epithelial cells. Due to the low amount of lavage fluid available in this paediatric population, we were able to obtain sufficient total RNA from the BALF cell pellet in only nine out of 49 patients. All patients displayed IgA class recombination and IgG1 class recombination was detected in two of these nine patients. This provides additional evidence for the local nature of the measured immunoglobulin production.

Lung function in $\mathrm{CF}$ is characterised by chronic structural damage leading to a decline in lung function. Impairment of lung function is the result of a combination of obstructive respiratory disease due to inflammation, mucus impactions and restrictive lung disease due to bronchiectasis and tissue destruction. Thus, local IgE to A. fumigatus may correlate with a reduced lung function. ABPA is a complication occurring in $\sim 9 \%$ of $\mathrm{CF}$ patients [8] linked to a hypersensitivity reaction to A. fumigatus. Basophil activation by $A$. fumigatus correlates with the clinical phenotype [16], and clinical improvement after anti-IgE and antimycotic treatment indirectly suggests a role of $\operatorname{IgE}$ and A. fumigatus in this context. However, specific IgE in the blood has not been proven to be a very good marker to predict ABPA and other immunoglobulin subclasses have been implicated in ABPA as well.
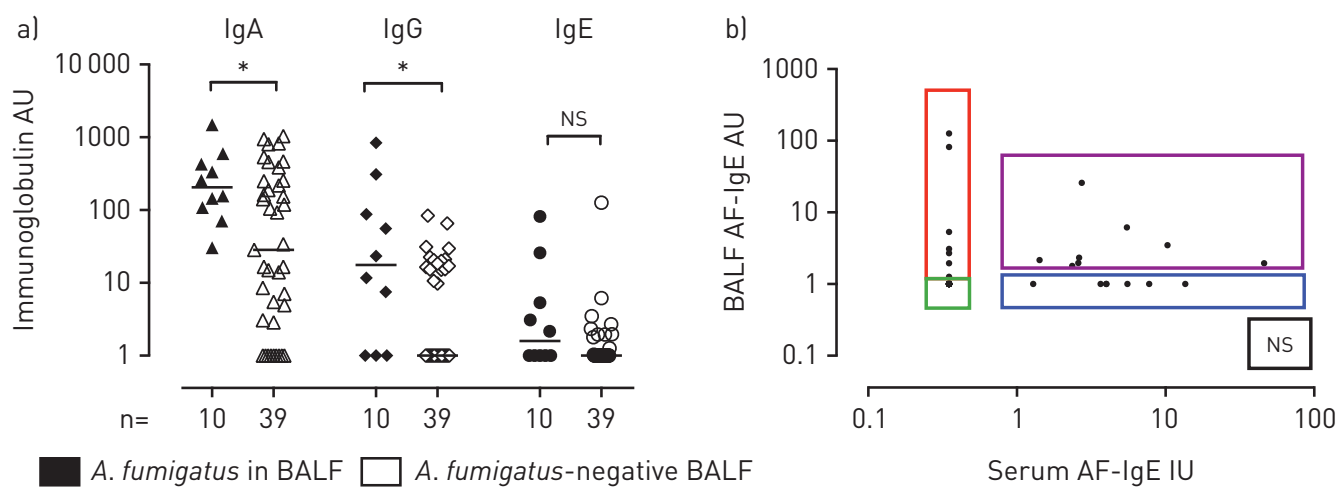

A. fumigatus in BALF $\square$ A. fumigatus-negative BALF

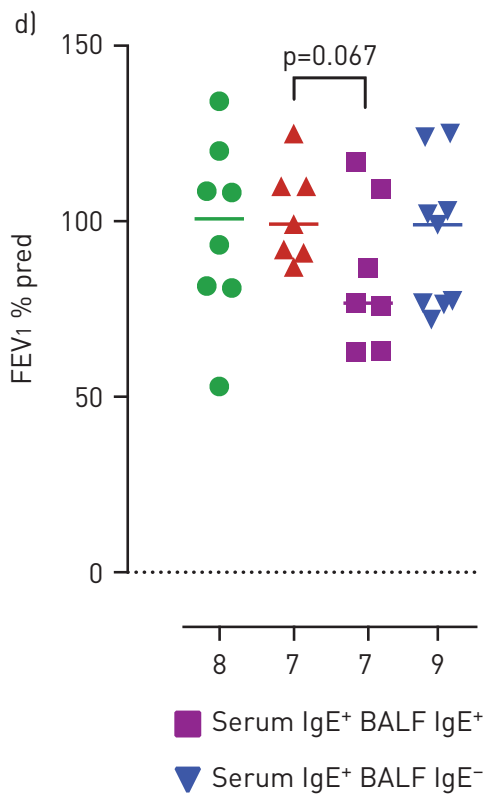

FIGURE 1 a) In the presence of Aspergillus fumigatus, local IgA and IgG levels to $A$. fumigatus are significantly elevated; *: $p<0.05$. b-d) Exclusive IgE production in the lung was associated with a significantly higher lung function as compared to patients that had serum and bronchoalveolar lavage fluid (BALF) A. fumigatus-specific $(A F)-\lg E(p<0.05$, Mann-Whitney U-test). FVC: forced vital capacity; FEV1: forced expiratory volume in $1 \mathrm{~s}$. 
To assess a potential impact of lung AF-IgE on lung function, patients were subgrouped based on local and systemic AF-IgE (BALF AF-IgE ${ }^{-} /$serum AF-IgE ${ }^{-}$, BALF AF-IgE ${ }^{+} /$serum AF-IgE $^{-}, \mathrm{BALF} \mathrm{AF}_{\mathrm{IgE}}^{+} /$ serum $\mathrm{AF}-\mathrm{IgE}^{+}$and $\mathrm{BALF} \mathrm{AF}-\mathrm{IgE}^{-} /$serum $\mathrm{AF}-\operatorname{IgE}^{+}$). Interestingly, the group of patients that had undetectable serum AF-IgE but a positive local response had a significantly higher forced vital capacity than CF patients with serum AF-IgE. Although forced expiratory volume in $1 \mathrm{~s}$ was not statistically significantly higher $(\mathrm{p}=0.067)$, this may be due to the small sample size (figure $1 \mathrm{c}$ and $\mathrm{d}$ ). Aspergillus is known to have potent type 2-inducing properties. This is considered to contribute to the pathogenicity of the fungus. Together with the reduced ability of CF patients to clear pathogens [17], this intrinsic type 2 -favouring action has been considered the reason for the high rates of sensitisation to A. fumigatus in CF patients. However, immune responses restricted to the local sites with no serum AF-IgE production may be a reflection of isolated local immune responses with a lack of atopic representation and consequently, a more efficient repulsion of A. fumigatus and other pathogens, resulting in an improved lung function. This needs to be addressed in larger cohorts.

In conclusion, we provide data on local production of antibodies specific to A. fumigatus in response to the presence of fungal growth in the lung of CF patients. Consequently, local IgE is not correlated with serum AF-IgE and most interestingly restricted specific AF-IgE responses in BALF were associated with a better lung function.

Mia Goña-Höpler ${ }^{1,7}$, Birgit Pfaller $^{2,7}$, Jonathan Argeny ${ }^{1}$, Stefan Kanolzer ${ }^{1}$, Saskia Gruber ${ }^{1}$, Klara Schmidthaler ${ }^{1}$, Sabine Renner ${ }^{1}$, Edith Nachbaur ${ }^{1}$, Petra Fucik ${ }^{1}$, Andreas G. Glaser ${ }^{3}$, Markus Debiasi ${ }^{1}$, Zsolt Szépfalusi ${ }^{1}$, Reto Crameri ${ }^{3}$, Claudio Rhyner ${ }^{3}$ and Thomas Eiwegger ${ }^{1,4,5,6}$

${ }^{1}$ Dept of Pediatrics and Adolescent Medicine, Medical University of Vienna, Vienna, Austria. ${ }^{2}$ Dept of Pediatrics, Division of Clinical Pharmacology and Toxicology, The Hospital for Sick Children, Toronto, Canada. ${ }^{3}$ Swiss Institute of Allergy and Asthma Research (SIAF), University of Zurich, Zurich, Switzerland. ${ }^{4}$ Division of Immunology and Allergy, Food allergy and Anaphylaxis Program, Dept of Paediatrics, The Hospital for Sick Children, Toronto, Canada. ${ }^{5}$ Translational Medicine, Research Institute, The Hospital for Sick Children, Toronto, Canada. ${ }^{6}$ Depts of Pediatrics and Immunology, The University of Toronto, Toronto, Canada. ${ }^{7}$ These authors contributed equally.

Correspondence: Thomas Eiwegger, Division of Immunology and Allergy, Food Allergy and Anaphylaxis Program, Dept of Paediatrics, Hospital for Sick Children, 555 University Ave, Toronto, Ontario, M5G 1X8, Canada. E-mail: thomas.eiwegger@sickkids.ca

Received: May 312017 | Accepted after revision: Sept 072017

Support statement: Supported by funds of the Oesterreichische Nationalbank (Anniversary Fund, project number 13846ONB) and the Medical Scientific Fund of the Mayor of the City of Vienna (number 11013). Work at SIAF was supported by Swiss National Science Foundation Grants 320030_149978/1 and 31NM_152038. Funding information for this article has been deposited with the Crossref Funder Registry.

Conflict of interest: None declared.

Acknowledgments: T. Eiwegger was involved in the conception, hypotheses delineation and design of the study. E. Nachbaur, Z. Szépfalusi, T. Eiwegger, J. Argeny, S. Kanolzer, K. Schmidthaler, P. Fucik, M. Goña-Höpler, R. Crameri, A.G. Glaser, C. Rhyner and M. Debiasi were involved in acquisition, analysis and interpretation of the data. M. Goña-Höpler and B. Pfaller were involved in analysis and writing the article. S. Renner, E. Nachbaur, Z. Szépfalusi and T. Eiwegger were involved in critical review of the manuscript.

\section{References}

1 Durham SR, Gould HJ, Thienes CP, et al. Expression of epsilon germ-line gene transcripts and mRNA for the epsilon heavy chain of IgE in nasal B cells and the effects of topical corticosteroid. Eur J Immunol 1997; 27: 2899-2906.

2 Oliveria JP, Salter BM, MacLean J, et al. Increased $\operatorname{IgE}^{+}$B cells in sputum, but not blood, bone marrow, or tonsils, after inhaled allergen challenge in subjects with asthma. Am J Respir Crit Care Med 2017; 196: 107-109.

3 Oliveria JP, Salter BM, Phan S, et al. Asthmatic subjects with allergy have elevated levels of IgE+ B cells in the airways. J Allergy Clin Immunol 2017; 140: 590-593.

4 Wilson DR, Merrett TG, Varga EM, et al. Increases in allergen-specific IgE in BAL after segmental allergen challenge in atopic asthmatics. Am J Respir Crit Care Med 2002; 165: 22-26.

5 van de Pol MA, Lutter R, van Ree R, et al. Increase in allergen-specific IgE and ex vivo Th2 responses after a single bronchial challenge with house dust mite in allergic asthmatics. Allergy 2012; 67: 67-73.

6 KleinJan A, Vinke JG, Severijnen LW, et al. Local production and detection of (specific) IgE in nasal B-cells and plasma cells of allergic rhinitis patients. Eur Respir J 2000; 15: 491-497.

7 Takhar P, Smurthwaite L, Coker HA, et al. Allergen drives class switching to IgE in the nasal mucosa in allergic rhinitis. J Immunol 2005; 174: 5024-5032. 
De Schryver E, Devuyst L, Derycke L, et al. Local immunoglobulin E in the nasal mucosa: clinical implications. Allergy Asthma Immunol Res 2015; 7: 321-331.

9 Gevaert $\mathrm{P}$, Nouri-Aria $\mathrm{KT}$, Wu H, et al. Local receptor revision and class switching to IgE in chronic rhinosinusitis with nasal polyps. Allergy 2013; 68: 55-63.

10 Van Zele T, Gevaert P, Holtappels G, et al. Local immunoglobulin production in nasal polyposis is modulated by superantigens. Clin Exp Allergy 2007; 37: 1840-1847.

11 Tiringer K, Treis A, Kanolzer S, et al. Differential expression of IL-33 and HMGB1 in the lungs of stable cystic fibrosis patients. Eur Respir J 2014; 44: 802-805.

12 Tiringer K, Treis A, Fucik P, et al. A Th17- and Th2-skewed cytokine profile in cystic fibrosis lungs represents a potential risk factor for Pseudomonas aeruginosa infection. Am J Respir Crit Care Med 2013; 187: 621-629.

13 Stevens DA, Moss RB, Kurup VP, et al. Allergic bronchopulmonary aspergillosis in cystic fibrosis - state of the art: Cystic Fibrosis Foundation Consensus Conference. Clin Infect Dis 2003; 37: Suppl. 3, S225-S264.

14 Moser M, Crameri R, Brust E, et al. Diagnostic value of recombinant Aspergillus fumigatus allergen I/a for skin testing and serology. J Allergy Clin Immunol 1994; 93: 1-11.

15 Nikolaizik WH, Moser M, Crameri R, et al. Identification of allergic bronchopulmonary aspergillosis in cystic fibrosis patients by recombinant Aspergillus fumigatus I/a-specific serology. Am J Respir Crit Care Med 1995; 152: 634-639.

16 Gernez Y, Walters J, Mirkovic B, et al. Blood basophil activation is a reliable biomarker of allergic bronchopulmonary aspergillosis in cystic fibrosis. Eur Respir J 2016; 47: 177-185.

17 Hartl D, Gaggar A, Bruscia E, et al. Innate immunity in cystic fibrosis lung disease. J Cyst Fibros 2012; 11: $363-382$. 